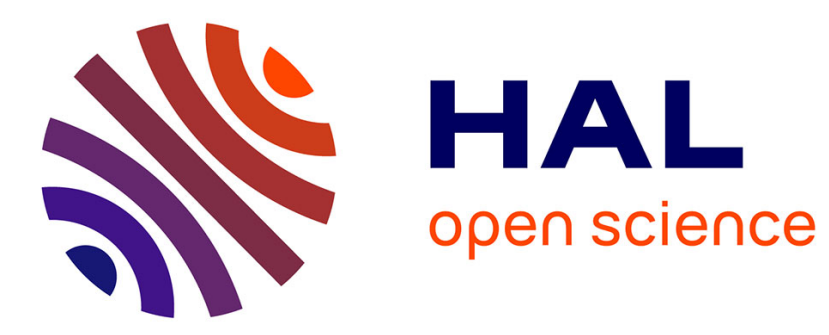

\title{
A robust multivariable approach for hybrid fuel cell supercapacitor power generation system
}

\author{
D. Hernandez, D. Riu, Olivier Sename, F. Druart
}

\section{To cite this version:}

D. Hernandez, D. Riu, Olivier Sename, F. Druart. A robust multivariable approach for hybrid fuel cell supercapacitor power generation system. European Physical Journal: Applied Physics, 2011, 54 (2), 10.1051/epjap/2011100252 . hal-00699210

\section{HAL Id: hal-00699210 https://hal.science/hal-00699210}

Submitted on 20 May 2012

HAL is a multi-disciplinary open access archive for the deposit and dissemination of scientific research documents, whether they are published or not. The documents may come from teaching and research institutions in France or abroad, or from public or private research centers.
L'archive ouverte pluridisciplinaire HAL, est destinée au dépôt et à la diffusion de documents scientifiques de niveau recherche, publiés ou non, émanant des établissements d'enseignement et de recherche français ou étrangers, des laboratoires publics ou privés. 


\title{
A robust multivariable approach for hybrid fuel cell supercapacitor power generation system
}

\author{
David Hernandez ${ }^{1}$, Delphine Riu ${ }^{1}$, Olivier Sename ${ }^{2}$ and Florence Druart ${ }^{3}$ \\ 1 G2Elab, Grenoble Electrical Engineering lab, Grenoble, France \\ 2 GIPSA-lab, Grenoble Images Parole Signal Automatique lab, Grenoble, France \\ 3 LEPMI, Laboratoire d'Electrochimie et de Physicochimie des Matriaux et des Interfaces, Grenoble, France
}

Received: date / Revised version: date

\begin{abstract}
In this article, a $\mathcal{H}_{\infty}$ control methodology is proposed for a hybrid power generation system composed by a $500 \mathrm{~W}$ PEM fuel cell and a $58 \mathrm{~F}$ supercapacitor. The control strategy consists in synthetizing a multivariable PI controller with $\mathcal{H}_{\infty}$ performance in order to manage powers between two electrochemical sources. The controller is then designed through an optimization procedure based on solving some Linear Matrix Inequalities (LMI). The control performance in time and frequency domains are then analyzed and compared with classical controllers. Results show the efficiency of the proposed methodology in order to reduce time spent for design.
\end{abstract}

\section{Introduction}

It is believed that fuel cells (FC) are expected to play a very importante role in the future of clean zero-emission power generation technologies. Nevertheless, several technological challenges in availability and hydrogen supply have to be overcome in the following years in order to develop this technology.

Most of time, fuel cells have to be associated to a storage device, like supercapacitor or battery, in order to mitigate harmful current transients and to increase its lifespan [13]. Classically, each component of the hybrid fuel cell control system is designed independently of each other. This approach allows simplifying the control strategy and design, but is not sufficient to take into account the several and various dynamics of the system and the coupling between thermodynamical (gas pressure for instance) and electrical (current, voltage or even power) variables. Besides, time spent for the design of such a complex system can become critical since it is often necessary to iterate for the calculation of controler parameters taking into account of all dynamic specifications.

This design is complexified as system parameters are not known accurately. Then, fuel cell performances are closely linked with temperature and membrane humidification. Moreover, stability of a DC system can be degraded thanks to interactions between converters. For that purpose, robustness methods seem to be particularly adapte since they are able to deal with control issues for uncertain systems.

Then, in this paper, classic multi-loop control approach used to add stability [1] [2] is compared with the proposed

Send offprint requests to: delphine.riu@g2elab.grenoble-inp.fr strategies presented in [3] and [4]. Besides, a good review on different electrical power conditioning structures for FC power generation is presented in [13]. [9] presents also a centralized controller for DC-DC power converter of a FC. However no storage is used, and then only time result simulations are presented and no robustness evaluation is performed. In parallel, control strategies often include FC energy management in order to optimize the efficiency of the FC [10][3]. The main objective of this paper is then to show that robustness allows to simplify and optimize the design of control loops for a hybrid system, and then come back over the design and improve the efficiency of the system.

In this article, the authors are interesed in the $\mathcal{H}_{\infty}$ robust control of a hybrid system composed by a $500 \mathrm{~W}$ PEMFC (Proton Exchange Membran Fuel Cell) with a 58 F supercapacitor (SC). Only electrical performances on the DC bus are considered. The thermodynamics control is then assumed to be perfect, with constant gas pressures. To cope with practical implementation constraints, a control strategy involving reduced order controllers is considered. The simplified method of the iterative Linear Matrix Inequalities (iLMI) algorithm, proposed by [11] and [12] for a PI controller, is used in this paper. Finally, a $\mu$-analysis is used for the robust control analysis. These methods are finally used to propose several control strategies and compare them with classical proposed in the literature.

This paper is then divided into four sections. In the first section, the studied hybrid system is presented and modelled for small-signal variations around the steadystate behaviour. Then, the classical control approaches are presented based on multi-loop control theory. The pro- 
posed control strategies are then detailed in the third section. Finally, the robust methodology is presented and applied on the hybrid system. Simulations in frequency and time domains allow to validate our approach.

\section{Modelling of studied system}

\subsection{System description}

The studied system configuration is presented in Figure 1. The hybrid system is composed by two sources and two parallel boost choppers without reversibility. The supercapacitor should be able to provide a sufficient instant power to guarantee normal operation in the presence of significative energy transients. A DC filter is also connected on the output in order to reject harmonic disturbances. The supercapacitor is recharged from the FC current with a Flyback chopper which is not represented on Figure 1.

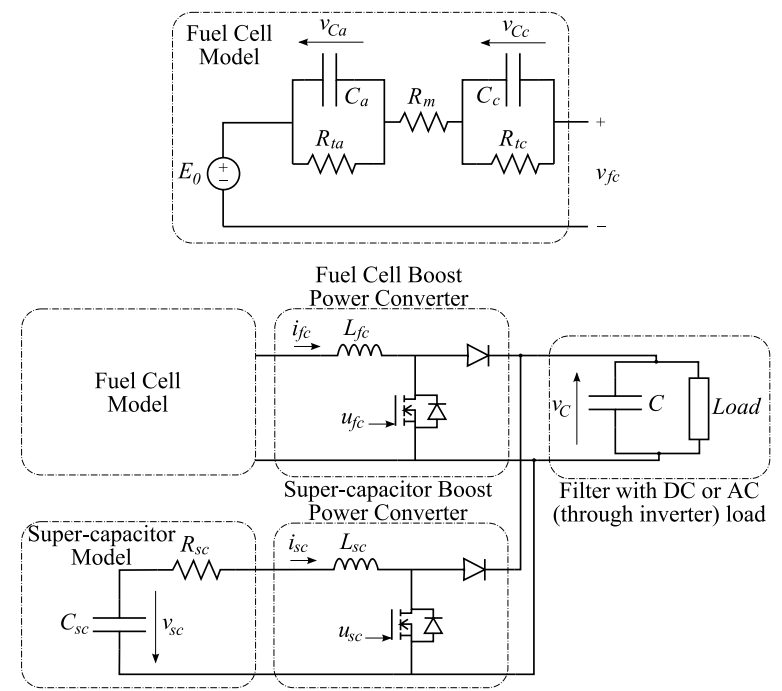

Fig. 1. Studied hybrid power generation system

\subsection{System modelling}

Relevant and accurate models have to be determined in order to guarantee good performances of the hybrid system in steady and transient states. This is espacially true for the fuel cell which is a complex and non linear component.

According to the complexity of the global system, a small signal approach has been prefered in order to use linear control theory. Then, for this study, equivalent electrical circuits are sufficient to model each component. Model parameters are then directly fitted from experimental data. In the case of fuel cell, static parameters are identified from the polarization curve of the fuel cell (see Figure 2). Dynamic parameters have been identified from Electrochemical Impedance Spectroscopy [5].

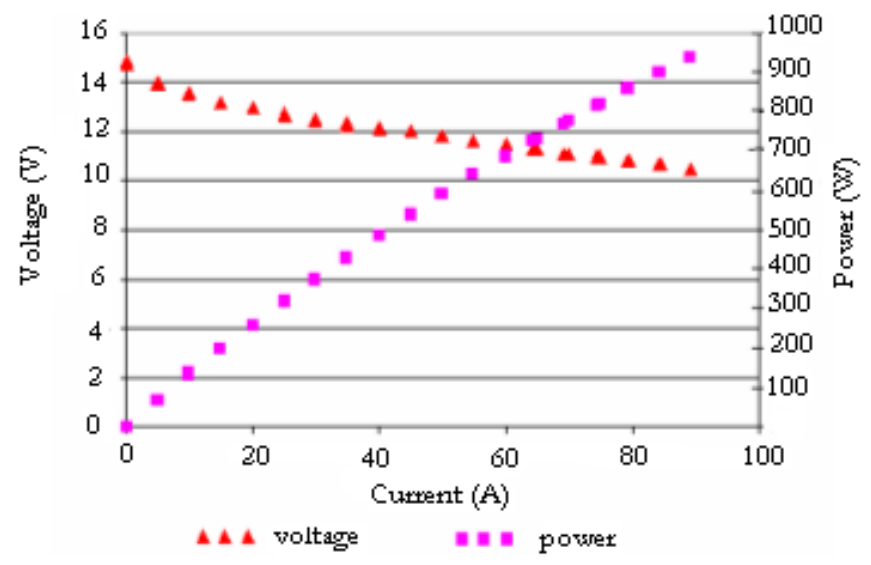

Fig. 2. Polarization curve of the studied PEM fuel cell for a gas overstoechiometry of 1.5

Table 1. FC/SC hybrid system parameters

\begin{tabular}{lll}
\hline Parameter & Value & Units \\
\hline \hline$E_{0}$ & 13.4 & Volts \\
$R_{m}$ & $1.28 \times 10^{-3}$ & $\Omega$ \\
$R_{t c}$ & $2.04 \times 10^{-3}$ & $\Omega$ \\
$R_{t a}$ & $4.72 \times 10^{-4}$ & $\Omega$ \\
$C_{a}=C_{c}$ & 2.12 & $\mathrm{~F}$ \\
$C_{s c}$ & 58 & $\mathrm{~F}$ \\
$R_{s c}$ & 0.019 & $\Omega$ \\
$L_{f c}=L_{s c}$ & 50 & $\mu \mathrm{H}$ \\
$C$ & 37.6 & $\mathrm{mF}$ \\
\hline \hline
\end{tabular}

Both energy sources are then modeled using equivalent electrical circuits. For the FC, a so-called double layer dynamic model is used [6]. The SC is modelled using a classical dynamic model composed with a (R-C) series circuit. The average modeling methodology, see [14] or [15], is then used to obtain a non-linear average model of the switching system, given by the following set of equations:

$$
\begin{gathered}
\frac{d V_{C}}{d t}=\frac{1}{C}\left[\left(1-\alpha_{f c}\right) I_{f c}+\left(1-\alpha_{s c}\right) I_{s c}-i_{\text {load }}\right] \\
\frac{d I_{f c}}{d t}=\frac{1}{L_{f c}}\left[E_{0}-V_{C_{a}}-V_{C_{c}}-R_{m} I_{f c}-\left(1-\alpha_{f c}\right) V_{C}\right] \\
\frac{d I_{s c}}{d t}=\frac{1}{L_{s c}}\left[V_{s c}-R_{s c} I_{s c}-\left(1-\alpha_{s c}\right) V_{C}\right] \\
\frac{d V_{C_{c}}}{d t}=\frac{1}{C_{c}}\left[I_{f c}-\frac{V_{C c}}{R_{t c}}\right] \\
\frac{d V_{C_{a}}}{d t}=\frac{1}{C_{a}}\left[I_{f c}-\frac{V_{C a}}{R_{t a}}\right] \\
\frac{d V_{s c}}{d t}=\frac{1}{C_{s c}} I_{s c}
\end{gathered}
$$

Uppercases indicate average values. The system parameters are given in Table 1 and were taken from [4] for a 500W PEMFC with a nominal DC bus output voltage at $24 \mathrm{~V}$.

The linearized system equations are given in the statespace form: 
Table 2. Steady-state values of linear system

\begin{tabular}{ccccccc}
\hline Variable & $V_{C_{e}}$ & $I_{f c_{e}}$ & $I_{s c_{e}}$ & $V_{C a, C c_{e}}$ & $\alpha_{f c_{e}}$ & $\alpha_{s c_{e}}$ \\
\hline Value & $24 \mathrm{~V}$ & $36.7 \mathrm{~A}$ & $0 \mathrm{~A}$ & $0.21 \mathrm{~V}$ & 0.46 & 0.38 \\
\hline
\end{tabular}

$$
\begin{gathered}
\Delta \dot{x}=A \Delta x(t)+B_{1} \Delta \omega(t)+B_{2} \Delta u(t) \\
\Delta z(t)=C_{1} \Delta x(t)+D_{11} \Delta \omega(t)+D_{12} \Delta u(t) \\
\Delta y(t)=C_{2} \Delta x(t)+D_{21} \Delta \omega(t)
\end{gathered}
$$

where:

$$
\begin{aligned}
& x=\left[\begin{array}{llllll}
V_{C} & I_{f c} & I_{s c} & V_{C_{c}} & V_{C_{a}} & V_{s c}
\end{array}\right]^{T} \\
& \Delta \omega=\left[i_{\text {load }}\right]^{T} \\
& \Delta u=\left[\begin{array}{ll}
\alpha_{f c} & \alpha_{s c}
\end{array}\right]^{T} \\
& A=\left[\begin{array}{cccccc}
0 & \frac{\left(1-\alpha_{f c_{e}}\right)}{C} & \frac{\left(1-\alpha_{s c_{e}}\right)}{C} & 0 & 0 & 0 \\
-\frac{\left(1-\alpha_{f c_{e}}\right)}{L_{f c}} & -\frac{R_{m}}{L_{f c}} & 0 & \frac{1}{L_{f c}} & \frac{1}{L_{f c}} & 0 \\
-\frac{\left(1-\alpha_{s c_{e}}\right)}{L_{s c}} & 0 & -\frac{R_{s c}}{L_{s c}} & 0 & 0 & \frac{1}{L_{s c}} \\
0 & \frac{1}{C_{c}} & 0 & -\frac{1}{R_{t c} C_{c}} & 0 & 0 \\
0 & \frac{1}{C_{a}} & 0 & 0 & -\frac{1}{R_{t a} C_{a}} & 0 \\
0 & 0 & \frac{1}{C_{s c}} & 0 & 0 & 0
\end{array}\right] \\
& B_{1}=\left[\begin{array}{llllll}
-\frac{1}{C} & 0 & 0 & 0 & 0 & 0
\end{array}\right]^{T} \\
& B_{2}=\left[\begin{array}{cccccc}
-\frac{I_{f c_{e}}}{C} & \frac{V_{C_{e}}}{L_{f c}} & 0 & 0 & 0 & 0 \\
-\frac{I_{s c_{e}}}{C} & 0 & \frac{V_{C_{e}}}{L_{s c}} & 0 & 0 & 0
\end{array}\right]^{T}
\end{aligned}
$$

matrices $C_{1}, C_{2}, D_{11}, D_{12}$ and $D_{21}$ will be specified later and depends on the chosen performance $z(t)$ and measured $y(t)$ outputs for the proposed control strategies. Subscript $e$ indicates steady-state equilibrium point. These values are given in Table 2 . The prefix $\Delta$ is from now on dropped for notation simplicity.

\section{Classical control strategies}

In this section, the load current in system 1 is modeled as $i_{\text {load }}=V_{C} / R_{L}$. Two classical multi-loop control approaches are presented.

\subsection{Control Strategy proposed by Valero et al [3]}

The classic control approaches considered in [3] are based on multi-loop control. Multiple feedback control strategies are used to solve the stability problems associated with boost converter control [1]. A single current loop is used for the $\mathrm{FC}$ boost converter. The $\mathrm{SC}$ boost converter is chosen to control the DC bus voltage with a square output voltage loop and a second stabilizing current loop. This structure is shown in Figure 3. The mean values of output variables $u_{f c}$ and $u_{s c}$ are equal to $\alpha_{f c}$ and $\alpha_{s c}$ respectively.

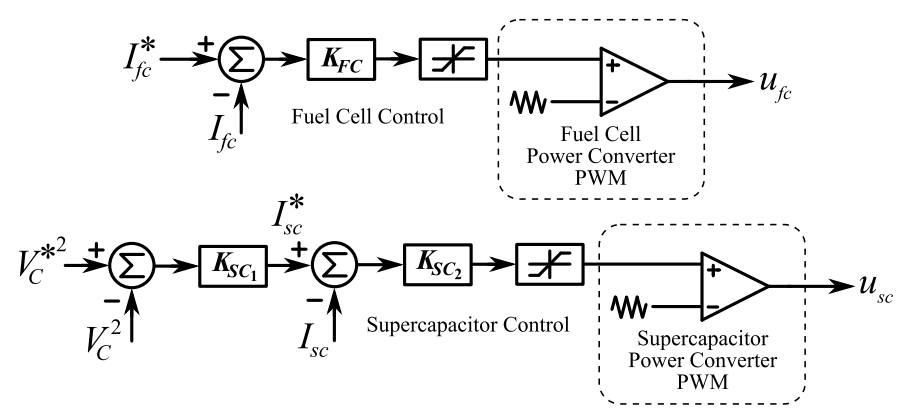

Fig. 3. Hybrid system control strategy proposed in [3].

Simple PI controllers of the form $K_{p}\left(1+K_{i} / s\right)$ are used for $K_{F C}$ and $K_{S C_{1}}$. The controller for $K_{S C_{2}}$ is chosen as a simple gain to assure rapidity of current control. The PI parameters are computed using pole compensation approaching a second order closed loop transfer function. Usually the second-order dynamics are defined by the damping ratio $\zeta$ and the undamped natural frequency $\omega_{n}$. These parameters are obtained by means of classic time domain performance parameters as the maximum overshoot, settling time, etc. The system equations are simplified to a first order transfer functions for each source.

The transfer functions for the $\mathrm{FC}$ and the $\mathrm{SC}$ are given respectively by:

$$
\begin{aligned}
\frac{I_{f c}}{\alpha_{f c}} & =\frac{V_{C_{e}}}{L_{s_{f c}} s+R_{s_{f c}}} \\
\frac{I_{s c}}{\alpha_{s c}} & =\frac{V_{C_{e}}}{L_{s_{s c}} s+R_{s_{s c}}}
\end{aligned}
$$

From further analysis in of [3] and based on the structure in Figure 3, the controllers parameters for a desired settling time $t_{s}$ were chosen as:

- FC current loop $K_{F C}(s)$ :

$$
k_{p}^{F C}=\frac{1}{V_{C_{e}}}\left(\frac{6 L_{s_{f c}}}{t_{s}}-R_{s_{f c}}\right), k_{i}^{F C}=\frac{R_{s_{f c}}}{L_{s_{f c}}}
$$

- SC voltage loop $K_{S C_{1}}(s)$ :

$$
k_{p}^{S C_{1}}=\frac{1}{V_{s c_{e}}}\left(C \zeta_{v} \omega_{n_{v}}-\frac{1}{R_{L}}\right), k_{i}^{S C_{1}}=\frac{\omega_{n_{v}}^{2} C}{2 k_{p} V_{s c_{e}}}
$$

The gain controller $K_{S C_{2}}$ is chosen from a desired bandwith $\omega_{n_{i}}$ as:

$$
k_{p}^{S C_{2}}=\frac{\omega_{n_{i}} L_{s_{s c}}-R_{s_{s c}}}{V_{C_{e}}}
$$

Using the parameters in Table 1 and choosing $t_{s}=$ $5 \mathrm{msec}, \omega_{n_{i}}=1000 \mathrm{rad} / \mathrm{s}, \omega_{n_{v}}=100 \mathrm{rad} / \mathrm{s}$ and $\zeta_{v}=1$, the simulation results, obtained with the non-linear average model for a $5 \%$ voltage reference step at $t=1$ sec and a $50 \%$ disturbance step at $t=2 \mathrm{sec}$, are shown in Figures 4 and 5 . 


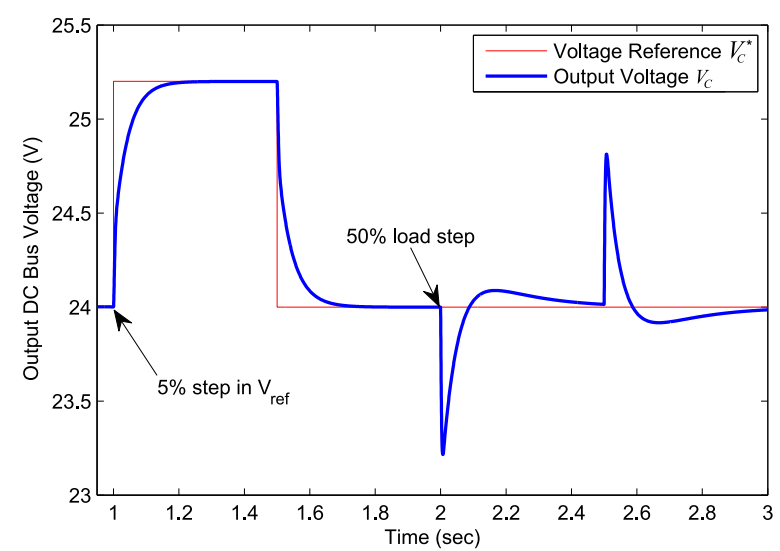

Fig. 4. Output voltage time response, method [3].

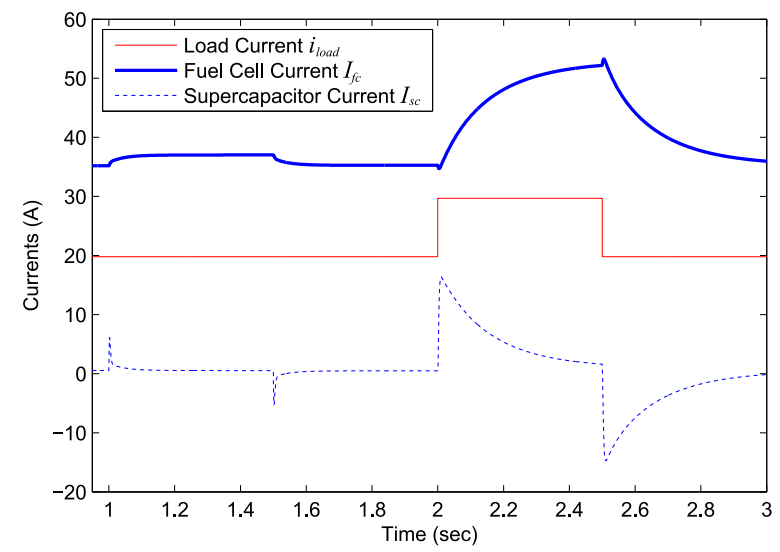

Fig. 5. System currents time response, method [3].

For this test case, the current reference $I_{f c}^{*}$ in Figure 3 is computed using an adapted filtered signal of the load current demand. The cut-off frequency of the filter is fixed at $1 \mathrm{~Hz}$. With this strategy the SC supplies the instantaneous transient current while the FC current has a smooth response to the disturbance, keeping a healthy FC operation as shown in Figure 5. Other constraints could be added to the FC dynamic, as the classic slope constraint with respect to the current density to avoid the so-called starvation problem [7].

\subsection{Control Strategy proposed by Sailler et al [4]}

A slightly modified version of the control strategy proposed in [4] is presented in Figure 6.

In this case, the FC control ensures the DC bus voltage control. Following the same procedure described before, the controller parameters are chosen as:

- FC voltage loop:

$$
k_{p}^{F C}=\frac{1}{V_{f c_{e}}}\left(C \zeta_{v} \omega_{n_{v}}-\frac{1}{R_{L}}\right), k_{i}^{F C}=\frac{\omega_{n_{v}}^{2} C}{2 k_{p} V_{f c_{e}}}
$$

For $\omega_{n_{v}}=480 \mathrm{rad} / \mathrm{s}$ and $\zeta_{v}=0.707$ the result shown in Figure 7 is obtained with the non-linear average model for a $5 \%$ voltage reference step at $t=1$ sec and a $50 \%$ disturbance step at $t=2$ sec.

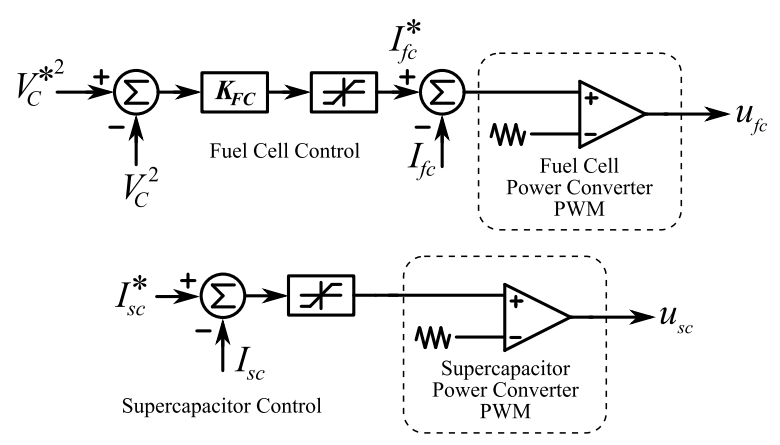

Fig. 6. Hybrid system control strategy proposed in [4].

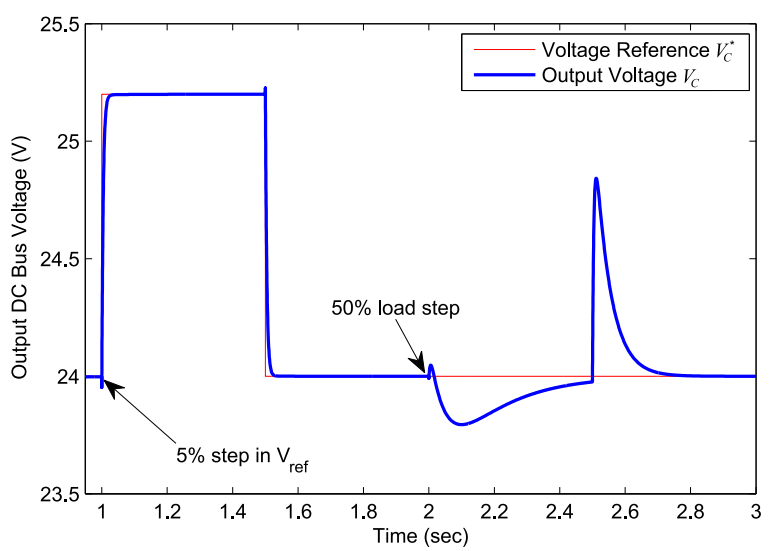

Fig. 7. Output voltage time response, method [4].

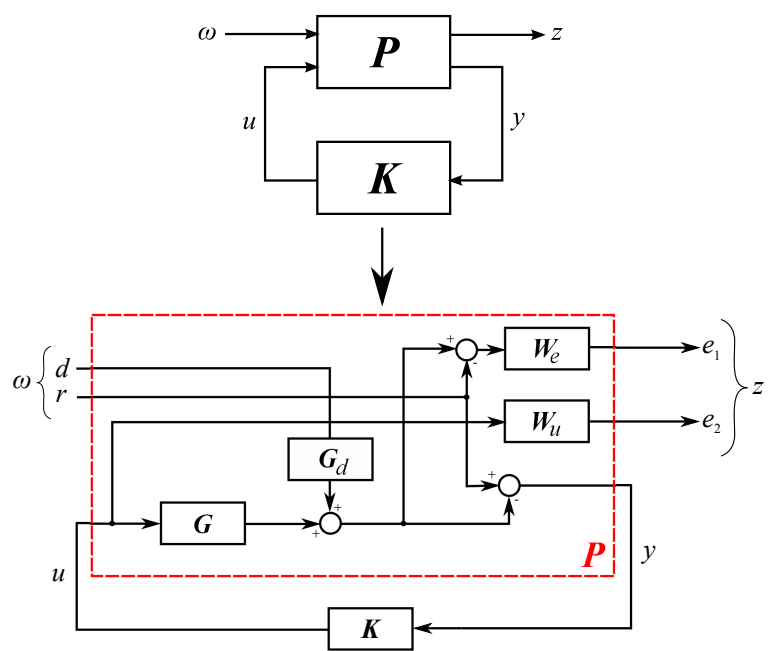

Fig. 8. General robust control configuration

\section{$4 \mathcal{H}_{\infty}$ Control strategies}

In this section, different robust control strategies proposed in this paper are reviewed. These control techniques are applied to the state-space system arranged in the general control configuration (shown in Fig. 8) and described by the set of equations 2 . In this paper, the authors are only interested in perturbation rejection, then reference $r$ is taken equal to 0 in the following section.

This linear model (2) can be extended to include the performance and robustness specifications, as done in the 
$\mathcal{H}_{\infty}$ approach. Particularly, some weighting functions can be included in the control design model in order to represent some templates on the sensitivity functions or to model the system uncertainties, as illustrated in [17].

The choice of these weighting functions will be developped later in sec. 5.1.

\subsection{System scalling}

Due to the important magnitude differences between several electric system components (capacitors, inductance and resistances for example), system scaling could be an important step before the control problem formulation. This will improve the conditioning of the problem and, thus, improve the optimizations algorithms performance. System scaling was performed in this paper using the prescale function in the MATLAB Control System Toolbox (Version 8.4 - Release 2009b). Inverse scaling of the controller to the original scale is not needed since output feedback control is considered.

\subsection{Multivariable PI control with $\mathcal{H}_{\infty}$ performance}

The proposed control strategy to design a multivariable PI controller, is based on solving some imposed LMI's constraints to the system (2) using the iterative algorithm proposed in [12]. The first step of this algorithm is a transformation so that the PI controller becomes a Static Output Feedback (SOF) controller. The SOF control problem, given some $\mathcal{H}_{\infty}$ performance criteria, is solved using the iLMI algorithm. The problem formulation of a PID controller into the SOF form is proposed in [11]. For this system (2) the problem formulation in the SOF form is to find a controller of the form $u(t)=F y(t)$, where $F \in \mathbb{R}^{m \times p}$ is such that the closed loop of the system satisfies certain desired performances.

For a multivariable PI controller, $u$ is given by:

$$
u(t)=F_{1} y(t)+F_{2} \int_{0}^{t} y(\theta) d \theta
$$

To impose a $\mathcal{H}_{\infty}$ performance, the controller in (5) should satisfy a closed loop transfer function $\left(T_{z \omega}\right)$ constraint of the form: $\left\|T_{z \omega}(s)\right\|<\gamma$ for $\gamma>0$. The $\mathcal{H}_{\infty}$ control problem of system (2)-(5) is given by the following LMI:

$$
\left[\begin{array}{ccc}
P A_{c l}+A_{c l}^{T} P & P B_{c l} & C_{c l}^{T} \\
B_{c l}^{T} & -\gamma I & D_{c l}^{T} \\
C_{c l} & D_{c l} & -\gamma I
\end{array}\right] \prec 0
$$

with: $A_{c l}=A+B_{2} F C_{2}, B_{c l}=B_{1}+B_{2} F D_{21}, C_{c l}=$ $C_{1}+D_{12} F C_{2}$ and $D_{c l}=D_{11}+D_{12} F D_{21}$.

(6) is a BMI (Bilinear Matrix Inequality), the algorithm proposed in [12] is then used to transform this into an iterative LMI problem. A simplified version of this algorithm and the SOF control formulation proposed in [11] for a PI controller is used in this paper. For this we note:

$$
\bar{x}=\left[\begin{array}{c}
x(t) \\
\int_{0}^{t} y(\theta) d \theta
\end{array}\right] \text { and } \bar{y}=\left[\begin{array}{c}
C_{2} x(t) \\
\int_{0}^{t} y(\theta) d \theta
\end{array}\right]
$$

Then the system composed by (2) and (5), setting $D_{21}=0$ without loss of generality, is given by:

$$
\begin{gathered}
\dot{\bar{x}}=\bar{A} \bar{x}(t)+\bar{B}_{1} \omega(t)+\bar{B}_{2} u(t) \\
z(t)=\bar{C}_{1} \bar{x}(t)+\bar{D}_{11} \omega(t)+\bar{D}_{12} u(t) \\
y(t)=\bar{C}_{2} x(t)
\end{gathered}
$$

with:

$$
\begin{gathered}
\bar{A}=\left[\begin{array}{cc}
A & 0 \\
C_{2} & 0
\end{array}\right], \bar{B}_{1}=\left[\begin{array}{c}
B_{1} \\
0
\end{array}\right], \bar{B}_{2}=\left[\begin{array}{c}
B_{2} \\
0
\end{array}\right] \\
\bar{C}_{2}=\left[\begin{array}{cc}
C_{2} & 0 \\
0 & I
\end{array}\right], \bar{C}_{1}=\left[\begin{array}{ll}
C_{1} & 0
\end{array}\right] \\
\bar{D}_{11}=D_{11}, \bar{D}_{12}=D_{12}
\end{gathered}
$$

The algorithm proposed by [12] is divided in two parts. In the first part an initial decision matrix $P$ is found through an iterative process. The iteration procedure is used to solve the BMI problem using the following set of linearizing variables:

$$
L=P^{-1}, V_{1}=P \bar{B}_{2} F, V_{2}=F \bar{C}_{2} L
$$

Variables $P, L, V_{1}$ and $V_{2}$ are used to compute the initial decision matrix $P_{i}$ according to the following simplified algorithm proposed by [12]. The second part of the algorithm computes the multivariable controller $F$.

\subsection{Full order $\mathcal{H}_{\infty}$ controller}

$\mathcal{H}_{\infty}$ control is considered in this paper as a mean to compare several robust control techniques based on $\mathcal{H}_{\infty}$ performance with the optimal complete order controller. $\mathcal{H}_{\infty}$ control synthesis is well known in literature. In this paper the LMI formulation for the $\mathcal{H}_{\infty}$ control is used to find a controller that satisfies the constraints in (6). The LMI formulation of this problem is well described in [16].

\subsection{Reduced order $\mathcal{H}_{\infty}$ controller}

A reduced order $\mathcal{H}_{\infty}$ controller is also considered for comparison. This type of controller is interesting because lowerorder controller design is allowed, which is important for real implementation. The computation of this controller is difficult because the LMI constraints that describe the control problem formulation usually leads to a nonsmooth optimization problem. This controller is in contrast with the full order $\mathcal{H}_{\infty}$ control, where the controller order equals that of the plant. To compute this controller a MATLAB Toolbox called hifoo has been developed by [18] and is used in this paper to solve the $\mathcal{H}_{\infty}$ norm minimization problem. 


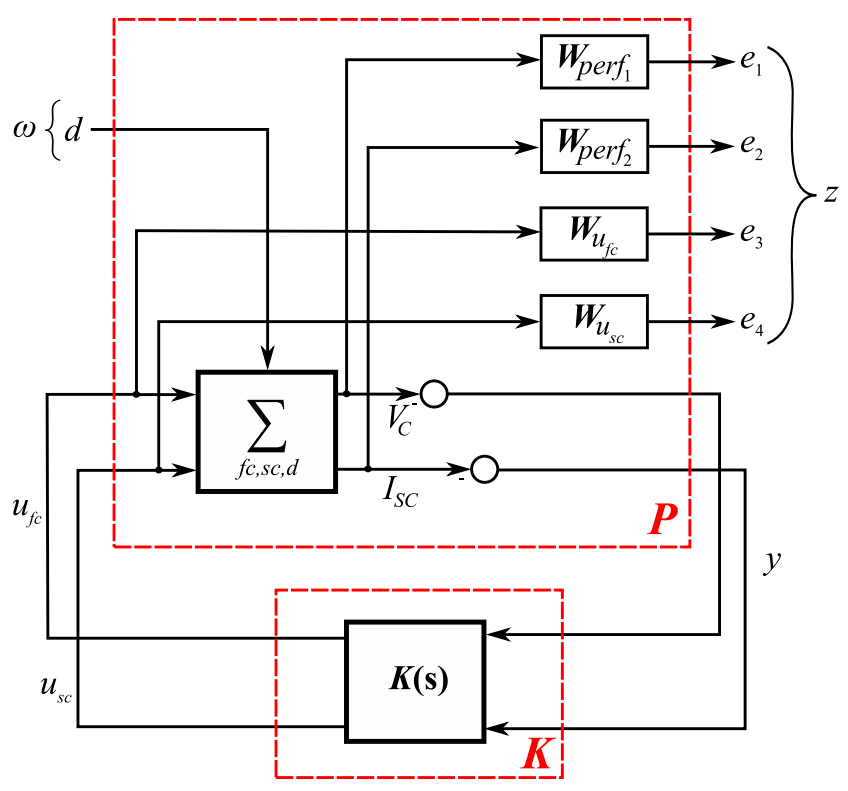

Fig. 9. Multivariable hybrid FC/SC system general control configuration.

\section{Proposed control design methodology}

\subsection{Control problem formulation}

The MIMO robust control synthesis is based on the general control configuration adapted to the hybrid $\mathrm{FC} / \mathrm{SC}$ system. The controlled outputs are the voltage $V_{C}$ and the SC current $I_{s c}$. The control objective is to keep a desired output voltage level and to control the SC current in order to provide the fast transient currents in the case of a load disturbance. Load transient rejection is important for the FC in order to avoid harmful operating conditions [19].

All controllers, including the classic PI control presented before, are designed to avoid exceeding normal operational output voltage ranges $( \pm 5 \%$ of rated output voltage is considered in this paper). In particular, the PI iLMI controller is designed to guarantee output voltage ranges and a stabilization time of $0.5 \mathrm{sec}$. The chosen configuration is shown in Fig. 8. The different weighting functions for this system, selected to guarantee the desired time/frequency performance described before, have been chosen as:

$$
\begin{aligned}
W_{\text {perf }} & =\frac{0.8 s+0.1}{s+0.001} \times I_{2} \\
W_{u_{1}} & =\frac{s+15.79}{0.01 s+15.71} \\
W_{u_{2}} & =\frac{s^{2}+3.947 \times 10^{5} s+6.2 \times 10^{6}}{0.01 s^{2}+3.927 \times 10^{5} s+6.169 \times 10^{4}}
\end{aligned}
$$

The iLMI algorithm yields a solution for the multivariable PI controller after five iterations, with a value of $\gamma=1.45 . \mathcal{H}_{\infty}$ controller for full and reduced order are also computed using the weighting functions presented before.

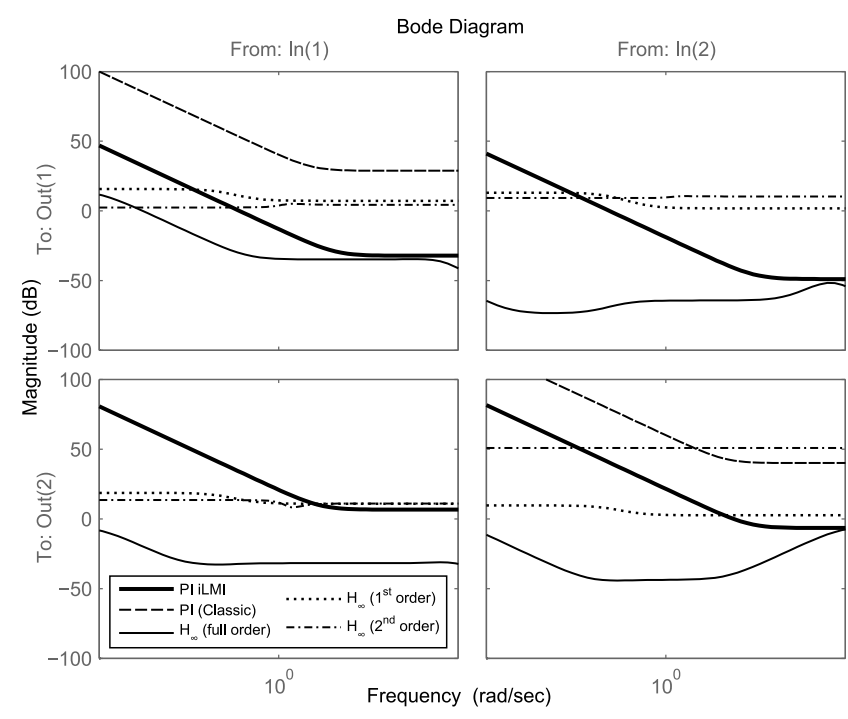

Fig. 10. Singular values plots of the different controllers.

The $\mathcal{H}_{\infty}$ full order controller found has 11 states and an optimal $\gamma=1.25$. The reduced order $\mathcal{H}_{\infty}$ controllers were computed after two iterations using the hifoo function with the $\mathcal{H}_{\infty}$ norm minimizing option.

The Bode diagrams of the different controllers are presented in Fig. 10. The frequency performance of the controllers indicate the importance of the MIMO controllers, when compared to the diagonal classic PI control.

The Bode diagrams of the MIMO sensitivity functions $S$ and $K S(s)$, are shown in Fig. 12 and Fig. 13 respectively. Results show how the shaping of $\mathcal{H}_{\infty}$ performance is straightforward for the PI iLMI and the full order $\mathcal{H}_{\infty}$ controllers using the templates weighting functions. These figures also show how performances are not entirely met using the reduced order $\mathcal{H}_{\infty}$ controllers, and the classical PI control. In contrast, the PI iLMI controller found, maintains a gain close to that of the optimal full order $\mathcal{H}_{\infty}$ controller, with a small resonant peak.

\subsection{Simulation results}

Simulation results for a $10 \%$ load step using the linearized closed loop system model, are shown in Fig. 12. The different controllers are compared. When compared, the settling times of the PI iLMI and the full order $\mathcal{H}_{\infty}$ controllers are bigger, this is done intentionally with the hope to obtain a better robustness, improving time/frequency trade-off. It should be noticed that low order $\mathcal{H}_{\infty}$ controlers are not able to fulfill performance specifications. Time response of the classic PI control is similar to that of the $1^{\text {st }}$ order $\mathcal{H}_{\infty}$ control and is not shown for the sake of simplicity.

Simulation tests and validations were also performed using the non-linear average and the complete topological (real) model of the studied system. It is worth noting that a second stabilizing current loop (multi-loop feedback) is needed when classic PI control is to be implemented. This is not required to obtain stable performance in the voltagemode control with the robust control strategies proposed 


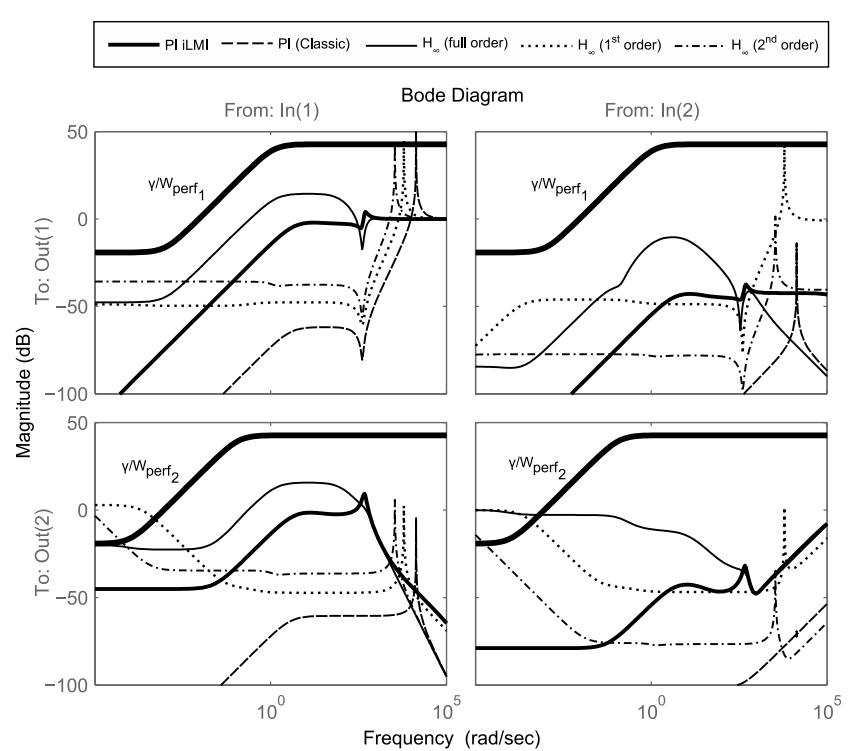

Fig. 11. Singular values plots for sensitivity functions $S_{1}$ and $S_{2}$.

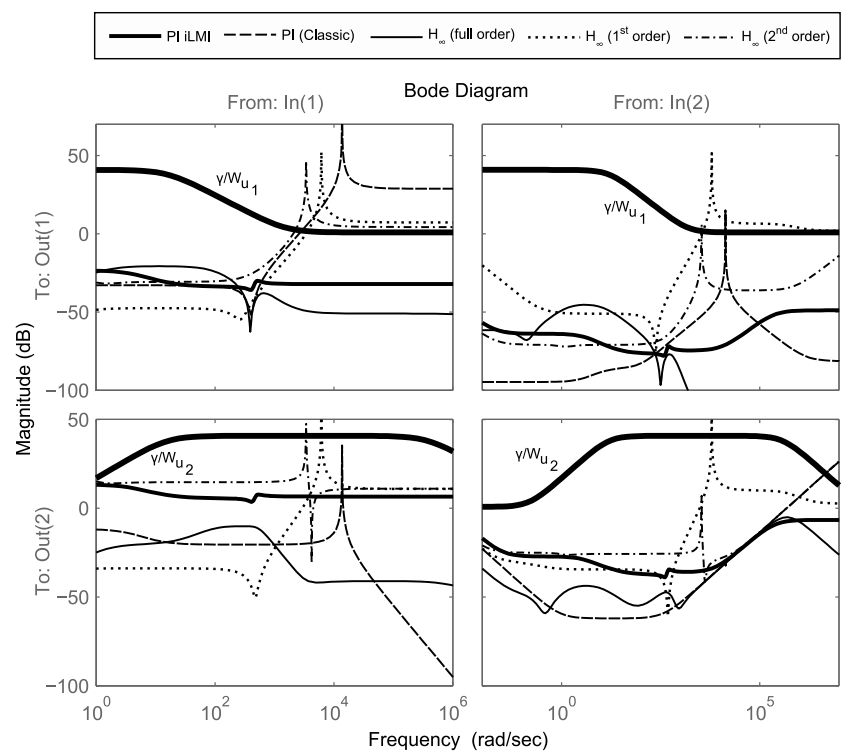

Fig. 12. Singular values plots for sensitivity functions $K_{1} S_{1}(s)$ and $K_{2} S_{2}(s)$.

in this paper. In the case of the PI iLMI control, the stability condition is included in the LMI formulation, so that stability is always guaranteed.

The PI and the full order $\mathcal{H}_{\infty}$ controllers are compared for validation using the non-linear average model of the studied system. Simulation results are shown in Fig. 14 for load steps of $10 \%$ at $t=2 \mathrm{sec}$ and $100 \%$ at $t=6 \mathrm{sec}$ respectively. The average non-linear model was validated using the complete topological model and the control strategies shown in Figs. ?? and 6. However, the stabilizing control loop was not necessary for the PI iLMI implementation. Results show the effectiveness of the proposed control, and control objectives are successfully achieved. The SC cur-

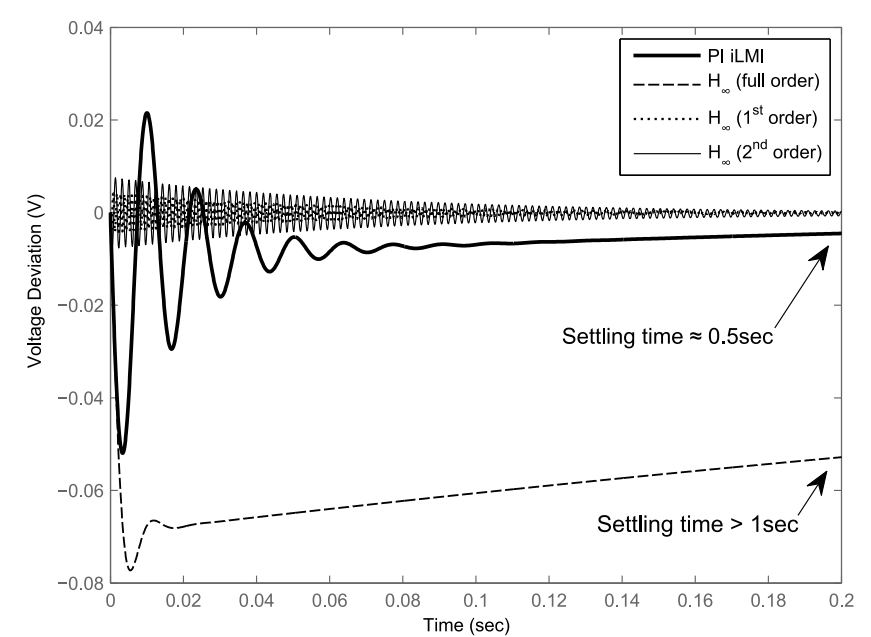

Fig. 13. Output voltage response to a load step disturbance.

rent contributes effectively to the load transient and output voltage is maintained within acceptable ranges.
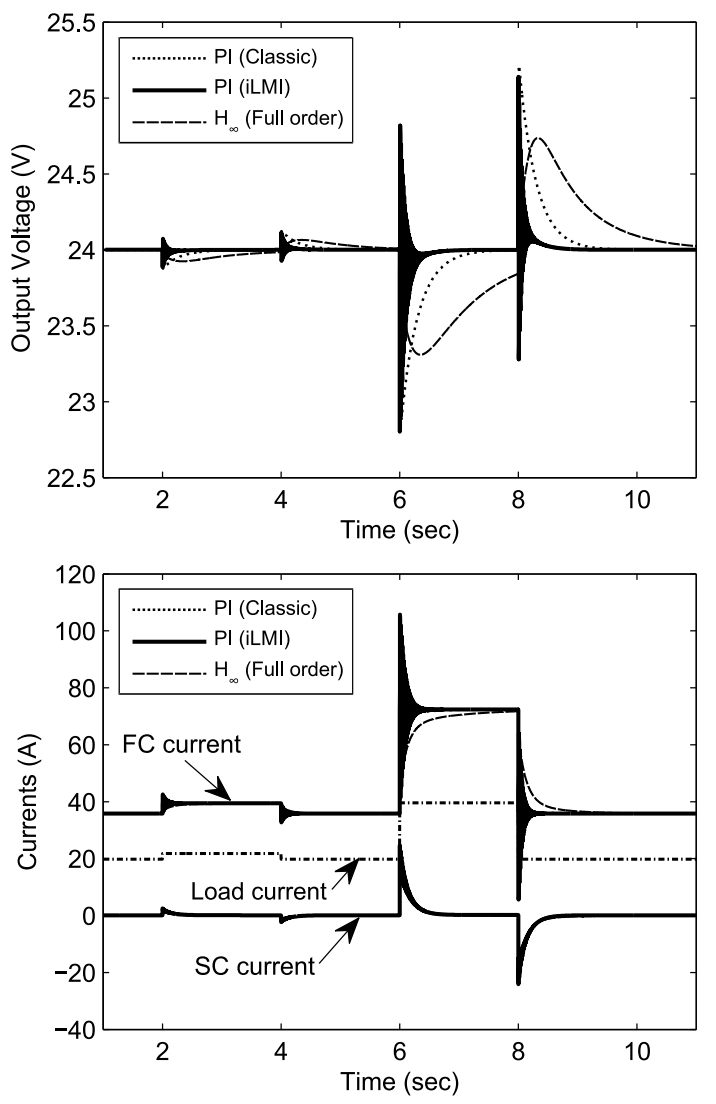

Fig. 14. Time simulation results using the complete non-linear average model.

As a comparison, time responses of classical strategies defined in [3] and [4] are represented on Fig. 15. Time responses indicate that robust controllers have better performances in disturbance rejection than classical approaches. Moreover, stability and robustness performances can not 
be guaranteed for these systems. Then in the next section, the robustness of controllers will be analysed only with $\mathcal{H}_{\infty}$ controllers of full or reduced orders.

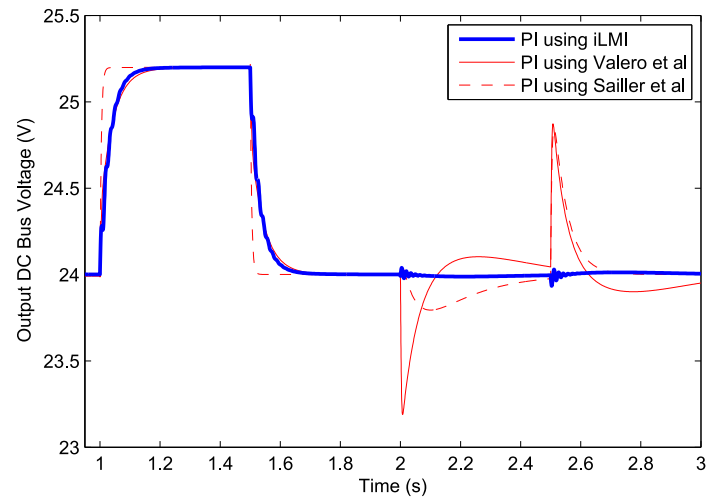

Fig. 15. Time simulation results using the complete non-linear average model.

\section{Robustness analysis}

The $\mathcal{H}_{\infty}$ performance control contribution relies on the controllers robustness improvement. The robustness analysis in the presence of model uncertainties is carried out using $\mu$-analysis. These techniques are detailed in [17], and are applied to practical problems in [6] and [20] for example. Uncertainties are modeled with the unstructured input-multiplicative form and are represented by the uncertainties weights. This configuration is presented in Fig. 16.

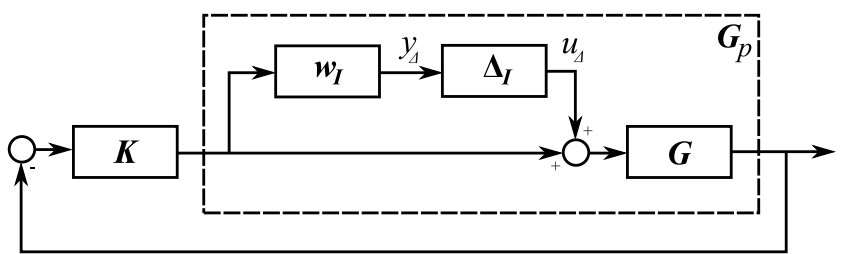

Fig. 16. Modeling uncertainties.

For the FC/SC hybrid system, the proposed configuration for $\mu$-analysis is shown in Fig. 17, where $N$ is the perturbed closed-loop system. The weighting functions for uncertainties modeling are computed using the ucover function in the MATLAB Control System Toolbox. These functions are computed plotting disturbance and control inputs for several model uncertainties. The weighting functions obtained and the sampled uncertain transfer functions of the system are presented in Fig. 18.

Robust stability and robust performance plots for parametric uncertainties of $10 \%$ in $C, C_{s c}, L_{f c}$ and $L_{s c}$, and of $20 \%$ in $R_{t a}, R_{t c}, R_{m}$ and $R_{s c}$, are presented in Figs. 19 and 20 .

From these results, the initial assumption of a better robustness performance using the PI iLMI and the

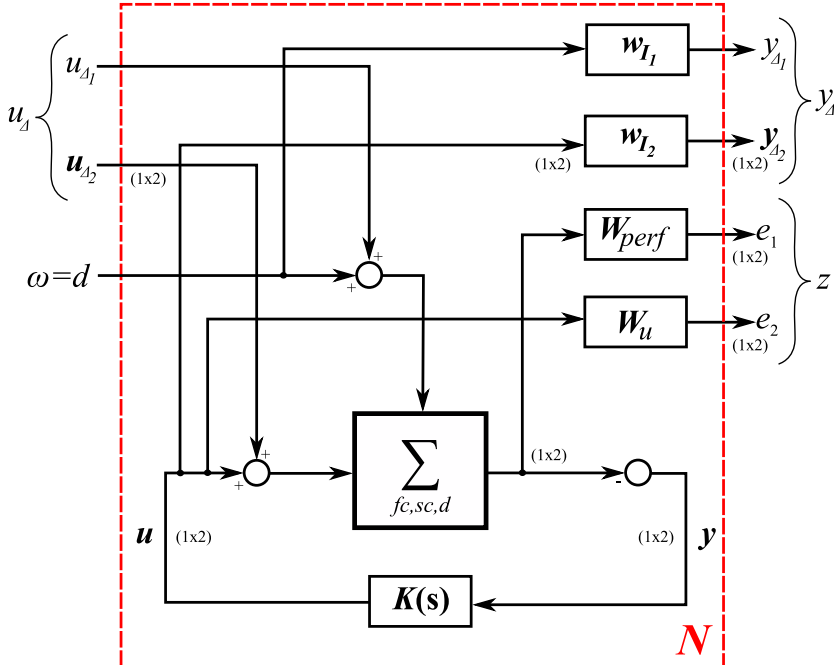

Fig. 17. Modeling uncertainties for the FC/SC hybrid system.
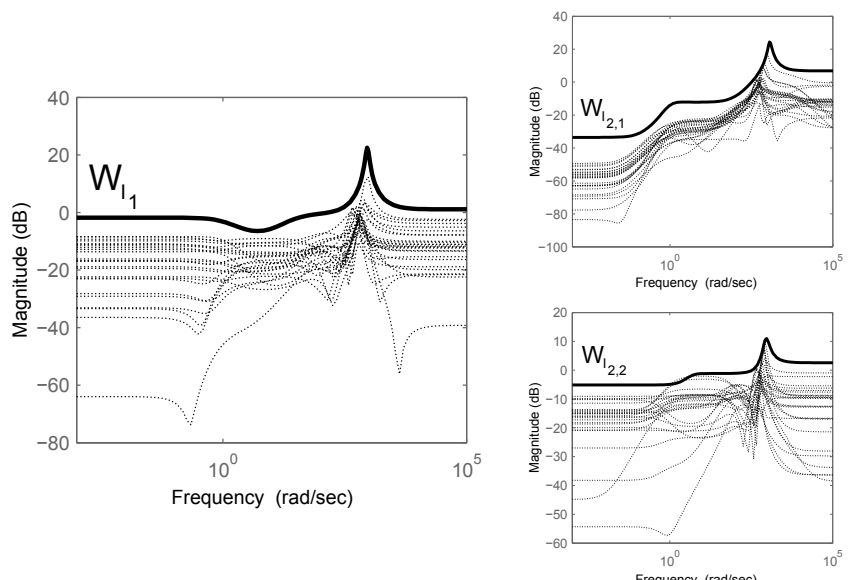

Fig. 18. Weighting functions for uncertainties modeling.

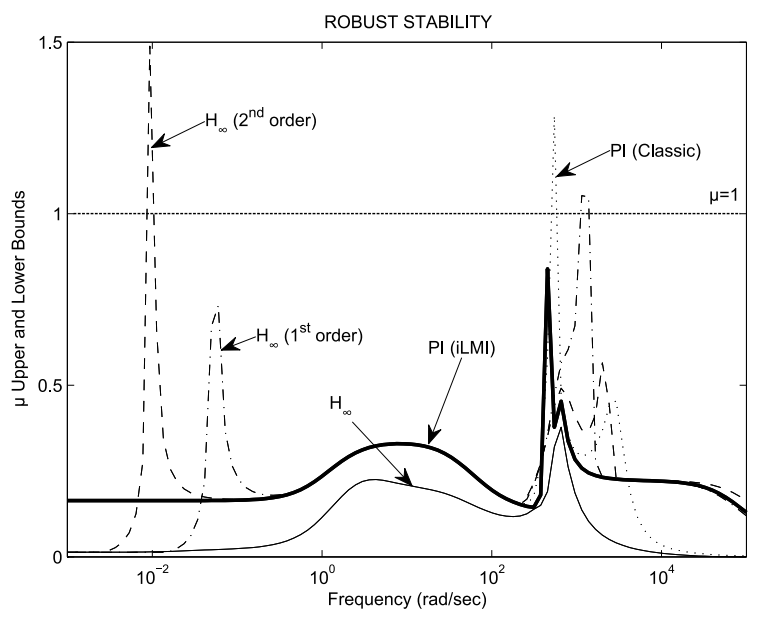

Fig. 19. Robust stability plot.

full order $\mathcal{H}_{\infty}$ is verified. The robust stability plots shows a maximum value of $\mu=0.8$ for the PI iLMI control. This means that the closed loop system remains stable with larger uncertainties of $(10 / 0.8) \%=12.5 \%$ for $C_{s c}$, 


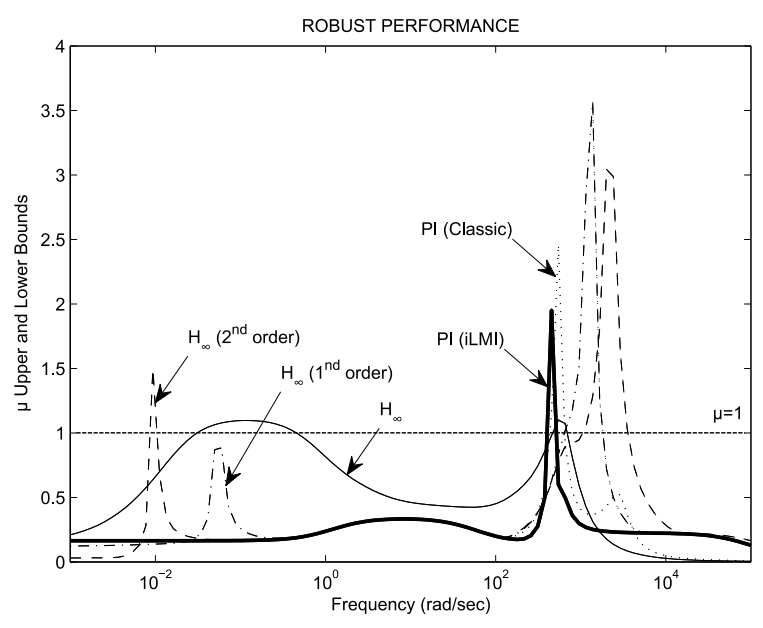

Fig. 20. Robust performance plot.

$L_{f c}$ and $L_{s c}$, and of $(20 / 0.8) \%=25 \%$ for $R_{t a}, R_{t c}, R_{m}$ and $R_{s c}$. For this uncertainty case we cannot conclude on the robust performance (RP), since $\mu=1.9>1$ for the PI iLMI control. However it is the more robust one after the full order $\mathcal{H}_{\infty}$ controller, which guarantees RP. For the other controllers RP is however satisfied with a lower uncertainty set, for example for $5 \%$ uncertainty in $C, C_{s c}, L_{f c}$ and $L_{s c}$, and of $10 \%$ in $R_{t a}, R_{t c}, R_{m}$ and $R_{s c}$. Robustness tests were also performed for uncertainties in the system load. Similar results were obtained in terms of controllers robustness. Robust stability is guaranteed for $110 \%$ uncertainty in the load modeled as a constant resistance $R$ using the PI iLMI control, this is not true for classic PI control. It should be noticed that $\mu$-analysis takes into account classical stability margins as gain and phase margins.

\section{Conclusion}

A complete time/frequency analysis, including a robustness analysis, has been performed on several robust control techniques. An important control approach has been proposed using simple MIMO PI control with stable $\mathcal{H}_{\infty}$ performance. Simulation results show the effectiveness of the proposed control strategy over classic PI tuning methods. This multivariable robust PI control could be developed as a generalized control design technique, improving time-consuming design procedures. The practical advantages of control robustness, on complex real problems, can be more easily addressed using the proposed methodology. The use of the generalized MIMO PI control methodology proposed in this paper can be extended to other several control strategies for power converters. Other control strategies could also include for example the effect of the hybrid power generation system connection to the utility network through an inverter. Multivariable control results could allow considering a more complet model of the FC system, including for example the thermodynamics model. Future experimental tests are envisaged for control validation, using the available Fuel Cell test-bench.

\section{References}

1. R.D. Middlebrook, IEEE Transactions on Power Electronics PE-2, (1987) 109-124.

2. J. Alvarez-Ramirez, I. Cervantes IEEE Transactions on Circuits and Systems 48-1, (2001) 103-106.

3. I. Valero, S. Bacha, E. Rullire, Journal of Power Sources 156-1, (2006) 50-56.

4. S. Sailler, F. Druart, D. Riu, P. Ozil, proceeding of 18th European Symposium on Computer Aided Process Engineering, (2008).

5. M. Usman, D. Riu, F. Druart, S. Rosini, Y. Bultel, N. Retire, journal of Power Sources, 160-2, (2006), 1170-1182.

6. I. Gadoura, T. Suntio, K. Zenger, proceedings of the IFAC World Congress on Automatic Control, (2002).

7. P. Thounthong, S. Rael, B. Davat, Journal of Power Sources 193-1, (2009) 376-385.

8. X. Yu, M. Starke, L. Tolbert, B. Ozpinecci, IET Electric Power Applications 1-5, (2007) 643-656.

9. K.W. Suh, A.G.Stefanopoulou, International Journal of Energy Research 29-12, (2005) 1167-1189.

10. Y. Song, S. Han, X. Li, S. Park, H. Jeong, B. Jung, Power Electronics Specialists Conference, (2007) 1261-1266.

11. F. Zheng, Q.G. Wang, T.H. Lee, Automatica 38, (2002) 517-526.

12. Y. He, Q.G. Wang, IEEE Transactions on Automatic Control 51, (2006) 1678-1683.

13. D. Hissel et al, Fundamentals and Developments of Fuel Cell conference, (2008).

14. R.D. Middlebrook, S. Cuk, International Journal of Electronics 42, (1977) 521-550.

15. S. Bacha, M. Brunello, A. Hassan, Electric Machines and Power Systems 22, (1994) 493-510.

16. C. Scherer, P. Gahinet, M. Chilali, IEEE Transactions on Automatic Control 42-7, (1997) 896-911.

17. S. Skogestad, I. Postlethwaite, Multivariable feedback control : analysis and design (Wiley and sons, New-York, 1996)

18. S. Gumussoy, D. Henrion, M Millstone, M. Overton, proceeding of IFAC Symposium on Robust Control Design, (2009).

19. C. Wang, M.H. Nehrir, IEEE Transactions on Energy Conversion 22, (2007) 864-872.

20. O. Sename, L. Dugard, Proceedings of European Control Conference, (2003).

21. D. Hernandez-Torres, M. Sautreuil, N. Retire, D. Riu, O. Sename, proceeding of IEEE ICIT conference, (2009), 166171. 